\title{
Eculizumab (ECU) Inhibits Thrombotic Microangiopathy (TMA) and I mproves Renal Function In Adult Patients (Pts) With Atypical Hemolytic Uremic Syndrome (aHUS)
}

Program: Oral and Poster Abstracts

Session: 101. Red Cells and Erythropoiesis, Structure and Function, Metabolism and Survival, Excluding Iron: Poster II

Sunday, December 8, 2013, 6:30 PM-8:30 PM

Hall E (Ernest N. Morial Convention Center)

Fadi Fakhouri ${ }^{1 *}$, Maryvonne Hourmant, MD $^{2 *}$, Spero $\mathrm{R}$ Cataland, M.D. ${ }^{3}$, Mario Espinosa $^{4 *}$, A. Osama Gaber ${ }^{5 *}$, Jan Menne ${ }^{6 *}$, Enrico Minetti ${ }^{7 *}$, Francois Provot ${ }^{8 *}$, Eric Rondeau $^{9 *}$, Piero Ruggenenti ${ }^{10^{*}}$, Laurent Weekers ${ }^{11^{*}}$, Masayo Ogawa, MD, FAAP ${ }^{12^{*}}$, Camille L. Bedrosian, MD ${ }^{12}$ and Christophe Legendre ${ }^{13^{*}}$

Introduction: aHUS is a rare, genetic, life-threatening disease of uncontrolled, chronic complement activation leading to TMA and severe end-organ damage. During the first clinical manifestation, end-stage renal disease (ESRD) or death occurs in approximately 33 to $40 \%$ of patients, and within one year of diagnosis, up to $65 \%$ have permanent renal damage, ESRD, or die despite receiving plasma exchanges/plasma infusions $(\mathrm{PE} / \mathrm{PI})$. Many aHUS pts are also misdiagnosed as TTP, which also results in poor outcomes with PE/PI. Hence, early diagnosis and effective treatment are critical to halt and/or prevent systemic complement-mediated damage. Ecu, a terminal complement inhibitor, is approved for the treatment of aHUS. Herein, we report safety and efficacy results of Ecu from the largest prospective study in aHUS to date.

Methods: This was an open-label, single-arm, Phase 2 trial of Ecu in adult aHUS pts. Inclusion criteria included platelet count $<150 \times 10^{9} / \mathrm{L}$ and $\mathrm{LDH} \geq 1.5$ ULN. Pts with STECHUS (shiga toxin + E. coli) and severe ADAMTS13 deficiency $(<5 \%)$ were excluded. An identified complement gene mutation was not required for admission. The primary endpoint was complete TMA response at 26 wks (normalization of platelets and LDH, and $\geq 25 \%$ improvement in serum creatinine from baseline (BL) on 2 consecutive measurements $\geq 4$ wks apart).

Results: 41 pts enrolled and 38 (93\%) received 26 wks of treatment (Table). 30 pts (73\%) were newly diagnosed, with a median of 2 wks to the first dose of Ecu. 6 pts (15\%) had no PE/PI during the current clinical manifestation. At wk 26, 30 pts $(73 \%)$ achieved complete TMA response, $36(88 \%)$ achieved hematologic normalization, and 40 (98\%) achieved platelet count normalization. Mean increase in platelet count from BL was $119 \times 10^{9} / \mathrm{L}(P<0.0001)$. Mean eGFR increase from $B L$ was $26.1 \mathrm{~mL} / \mathrm{min} / 1.73 \mathrm{~m}^{2}$ $(P<0.0001$ ). Platelet count (Fig 1) and eGFR (Fig 2) increased significantly from BL through wk 26. Of 24 pts on dialysis at BL, 20 (83\%) discontinued dialysis by wk 26 . Two pts not on dialysis at BL initiated and remained on dialysis through 26 wks. QoL significantly improved. Ecu was generally safe and well tolerated. Two pts had meningococcal infections, one of whom continued Ecu. No pts died.

Conclusions: Ecu normalized hematologic parameters and significantly improved renal function and QoL. The results of this prospective study confirm that Ecu inhibits complement-mediated TMA in adult aHUS pts, and point to the importance of early and accurate diagnosis, including ADAMTS13 testing. Due to high morbidity and mortality in aHUS despite PE/PI, recent guidelines recommend Ecu as first-line treatment in adults once an unequivocal diagnosis is made. The current data validate this treatment approach. This study is ongoing. 
Table: Baseline characteristics

\begin{tabular}{|c|c|}
\hline \multicolumn{2}{|l|}{ Baseline Demographics and Disease Characteristics $(N=41)$} \\
\hline Age, mean (SD), y & $40.3(15.3)$ \\
\hline Female sex, n (\%) & $28(68)$ \\
\hline Identified complement regulatory protein mutation or auto-antibody, n (\%) & $20(49)$ \\
\hline Time from aHUS diagnosis until screening (mo), median (range) & $0.8(0-311)$ \\
\hline Newly diagnosed pts, n (\%) & $30(73)$ \\
\hline Duration of current clinical manifestation of aHUS (mo), median (range) & $0.5(0.0-19.1)$ \\
\hline PE/PI during current clinical manifestation of aHUS, n (\%) & $35(85)$ \\
\hline Dialysis at BL, n (\%) & 24 (59) \\
\hline Prior renal transplant, n (\%) & $9(22)$ \\
\hline Plt count $<150 \times 10^{9} / \mathrm{L}, \mathrm{n}(\%)$ & $27(66)$ \\
\hline LDH >ULN, n (\%) & $32(78)$ \\
\hline $\mathrm{eGFR} \leq 60 \mathrm{~mL} / \mathrm{min} / 1.73 \mathrm{~m}^{2}, \mathrm{n}(\%)$ & $41(100)$ \\
\hline \multicolumn{2}{|l|}{ Efficacy Outcomes } \\
\hline Complete TMA response*, n (\%) & $30(73)$ \\
\hline 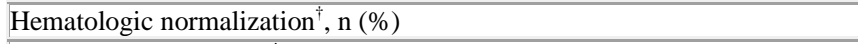 & $36(88)$ \\
\hline Plt count normalization ${ }^{\ddagger}, \mathrm{n}(\%)$ & $40(98)$ \\
\hline Plt count increase $\left(\mathrm{x} 10^{9} / \mathrm{L}\right)$, mean $(95 \% \mathrm{CI})$ & $\begin{array}{l}119(94 ; 145) \\
P<0.0001\end{array}$ \\
\hline eGFR increase from $\mathrm{BL} \geq 15 \mathrm{~mL} / \mathrm{min} / 1.73 \mathrm{~m}^{2}, \mathrm{n}(\%)$ & $22(54)$ \\
\hline eGFR increase from BL (mL/min/1.73 m²), mean (95\% CI) & $\begin{array}{l}26.1(19.8 ; 32.4) \\
P<0.0001\end{array}$ \\
\hline CKD improvement $\geq 1$ stage from BL, $\mathrm{n}(\%)$ & $26(63)$ \\
\hline
\end{tabular}

* Complete TMA response: normalization of plts and LDH, and $\geq 25 \%$ improvement in serum creatinine from BL on 2 consecutive measurements $\geq 4$ wk apart.

${ }^{\dagger}$ Complete hematologic response: plt and LDH normalization at $\geq 2$ consecutive measurements $\geq 4$ wk apart.

${ }^{\ddagger}$ Plt count normalization: plt count $\geq 150 \times 10^{9} / \mathrm{L} \geq 2$ consecutive measurements $\geq 4$ wk apart. 
Figure 1: Platelet count improvement through 26 weeks.

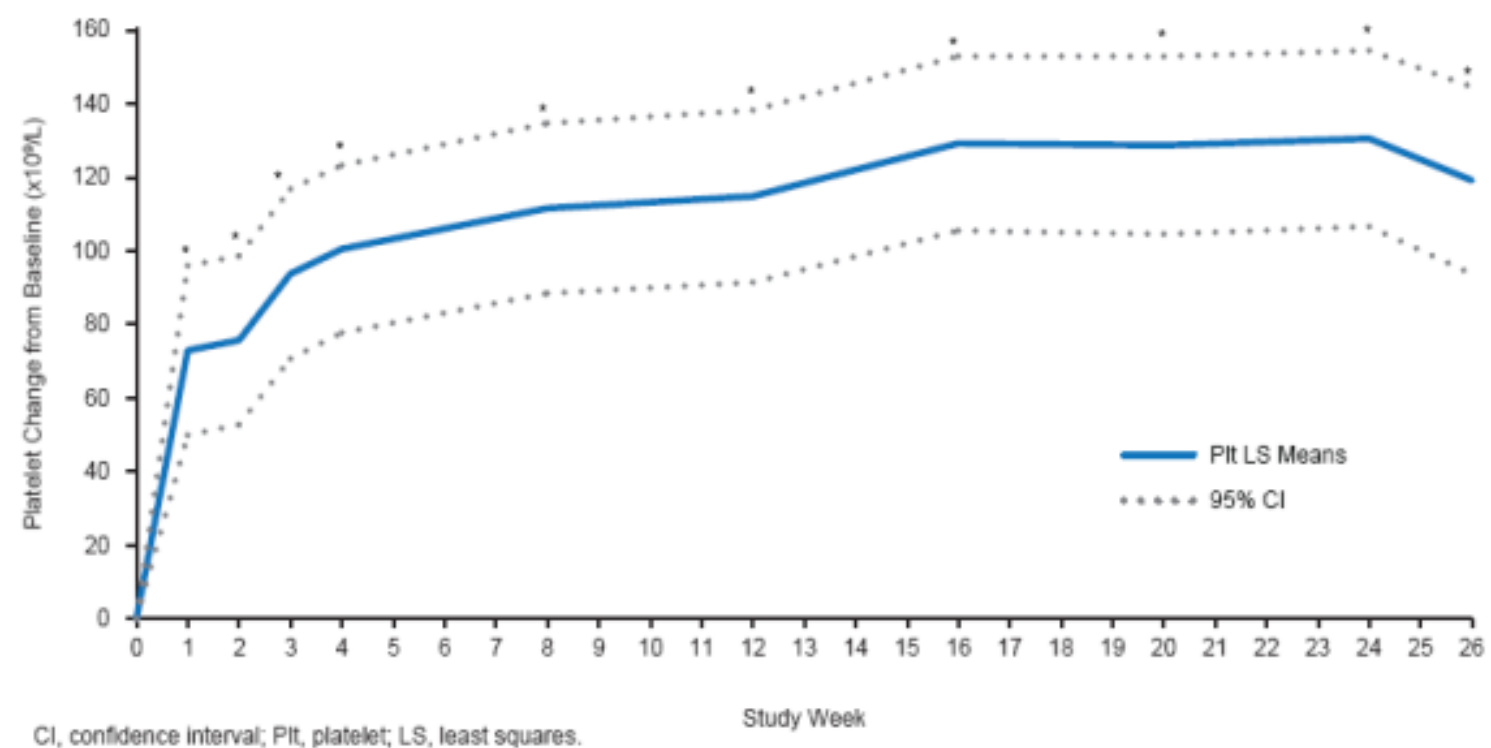
$\mathrm{N}>5$ shown. $* P=0.0001$.

$\mu$ 


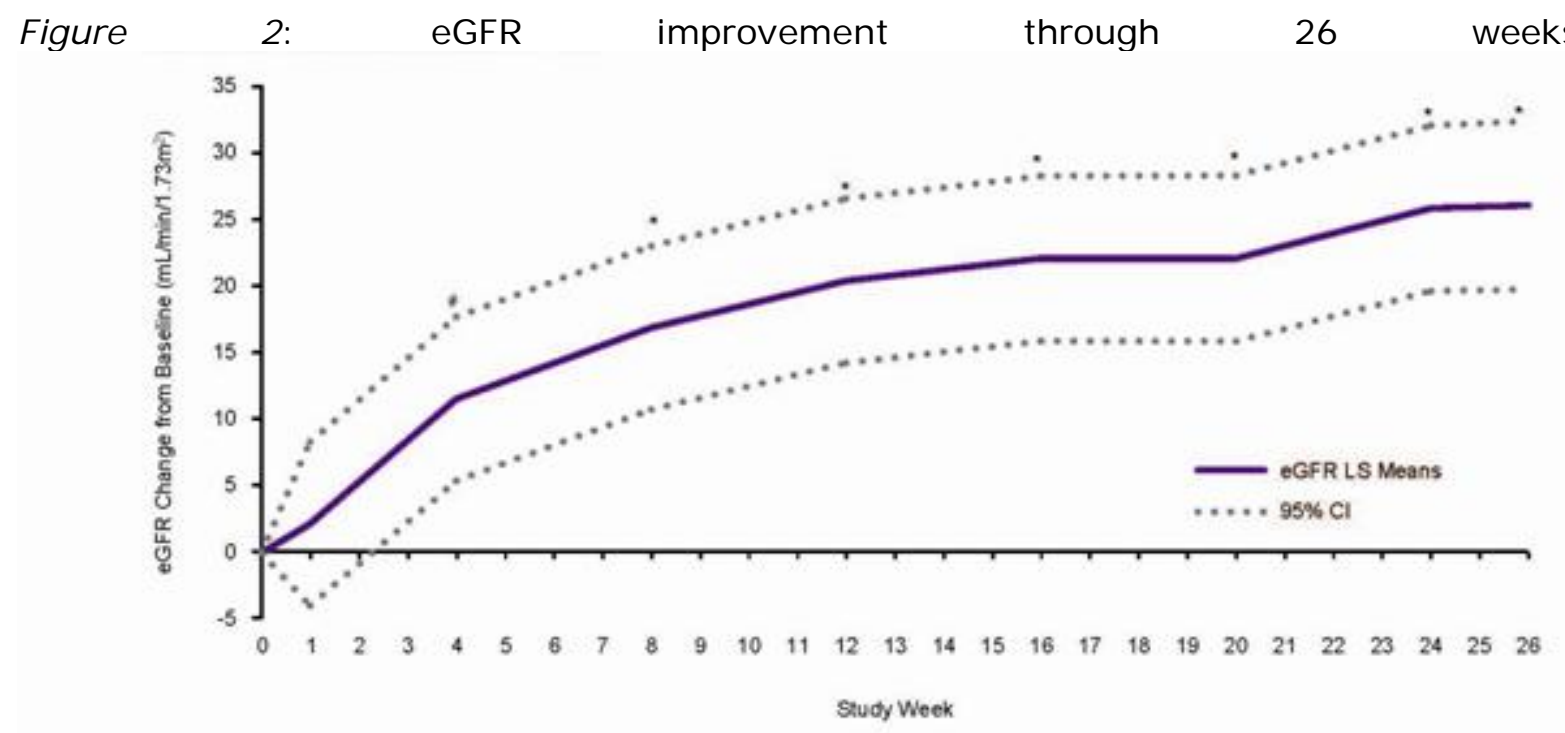

$\mathrm{Cl}$, confidence interval; eGFR, estimated glomerular filtration rate; LS, least squares. $N>5$ shown.

$* P<0.0001: * P<0.001$

Disclosures: Weekers: Roche, Novartis, Astellas: Grant Support Other, Research Funding. Ogawa: Alexion Pharmaceuticals: Employment. Bedrosian: Alexion Pharmaceuticals: Employment. 